\title{
Nueva especie de Mauria (Anacardiaceae) para Colombia
}

\section{New species Mauria (Anacardiaceae) for Colombia}

\author{
EnRiQue Renteria Arriaga*
}

\section{RESUMEN}

Dentro del proyecto realizado con patrocinio de Colciencias sobre el género Mauria de Colombia, se descubrieron varias especies, y hoy se publica una de esas especies nuevas., El tipo de polinización de la especie es entomófila, el síndrome que presenta sus poblaciones son árboles y arbustos hojas simples, espiraladas, elíptico-lanceoladas u obovadas, chartácea. Inflorescencia terminal o axilar, panicula, flores pequeñas, corola rosada, pétalos pequeños 5 , estambres 10, fruto oblongo, los granos de polen, son pequeños, oblado esferoidal, isopolar con simetría bilateral, tricolporado, la exina es estriada; nectario presentes, aromas fuertes. Su hábitat preferido son los lugares cerca de quebradas, fuentes de agua y zonas anteriormente bañadas por éstas y en pequeñas pendientes en suelos de cordilleras. El análisis del material disponible permite concluir que existen dos subespecies (Mauria amaliae Subespecie amaliae y Mauria amaliae subespecie huilense), presentes en Santa Marta y el Huila.

Palabras clave: Mauria amaliae Subespecie amaliae; Mauria amaliae subespecie huilense; Santa Marta; Huila; Anacardiaceae; Colombia.

\section{ABSTRACT}

Within the project with sponsorship Colciencias on gender Mauri of Colombia, were found several species that we want to publish one of these new species as a result of this research, the type of pollination of the species is entomofila, the syndrome which presents its populations are: trees and shrubs, leaves simple, spiral, elliptic-lanceolate or obovate, chartacea. Inflorescence terminal or axillary panicle, flowers small, corolla pink, 5 petals small, stamens 10 Fruto oblong pollen grains are small, spheroidal Oblada, isopolar with bilateral symmetry, tricolporado, the exine is striate; nectary present, strong aroma His preferred habitat are the places near streams, water sources and areas previously drenched them in small ridges of soil on slopes. The analysis of the material available to the conclusion that there are two subspecies. (Amaliae Subspecies amaliae Mauri, Mauri amaliae subspecies and Huila), in Santa Marta and Huila.

Keywords: Mauria amaliae Subespecie amaliae; Mauria amaliae subespecie huilense; Santa Marta; Huila; Anacardiaceae; Colombia.

\section{INTRODUCCIÓN}

El género Mauria Kunth es un grupo neotropical originario de los Andes compuesto de 25 especies. Su mejor representación se encuentra en Perú (Macbride, 1951) y Colombia (Rentería, 1988) con 15 y 14 especies respectivamente. La
* Grupo de Investigación en Biosistemática, Universidad Tecnológica del Chocó, Quibdó, Chocó, Colombia.

e-mail: enriquerenteria111@gmail.com enriquerenteria11@hotmail.com

Recibido: agosto 21, 2008

Aceptado: septiembre 10, 2008 


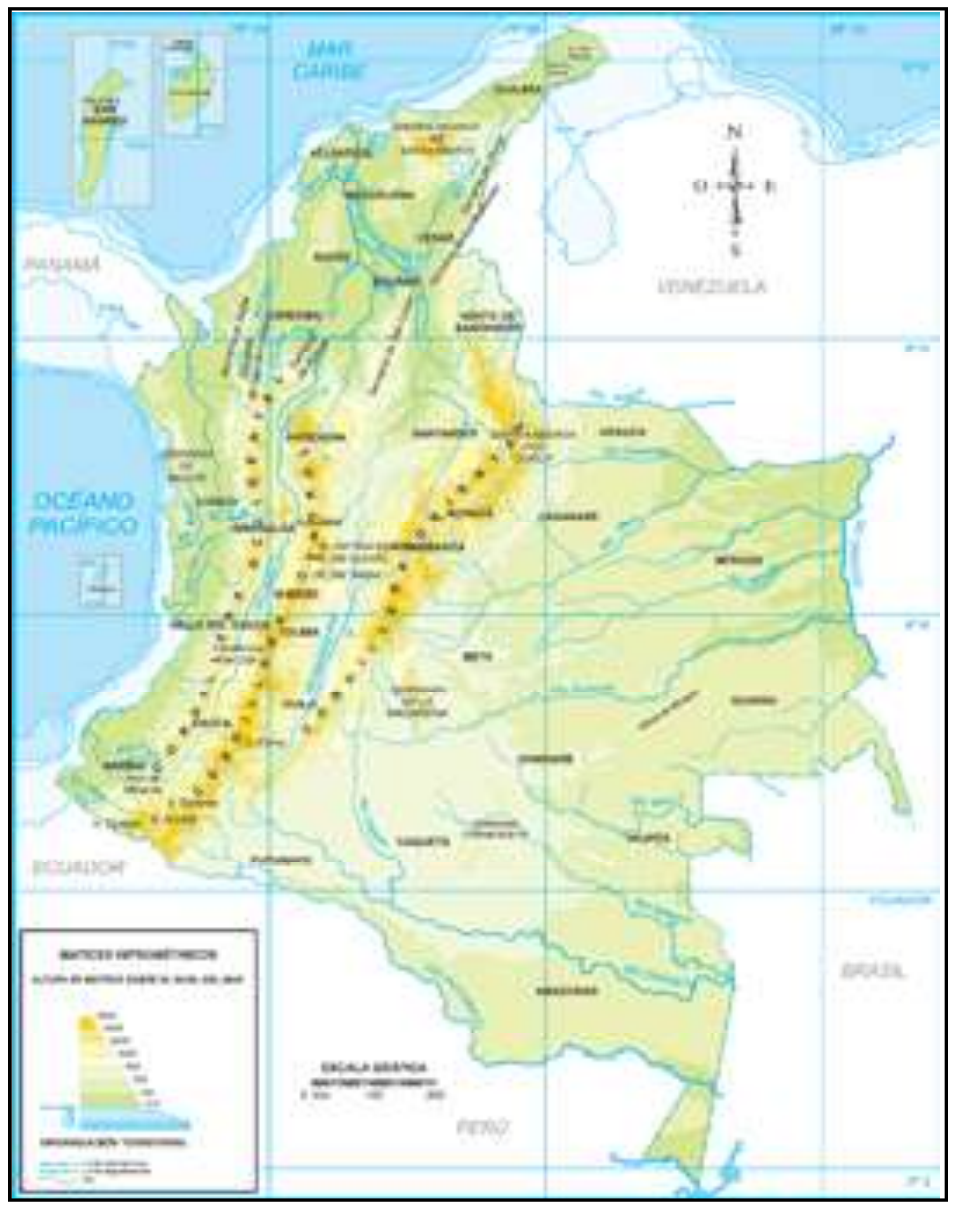

Figura 1. Distribución geográfica del género Mauria para Colombia, mostrando a M. amaliae Sierra Nevada y el Huila

familia Terebinthaceae, luego publicó dos especies Mauria simplicifolia y Mauria heterophylla (Kunth, 1825; Lindley 1831) lo incluyó en la tribu Sumachineae de la familia Anacardiaceae, caracterizada y delimitada táxonomicamente por él, pero considerada por Endlicher (1836) como tribu Anacardineae de las Terebintaceae. Después, Poeppig (1845), Tulasne (1846) y Turzaninow (1858) publicaron cinco nuevas especies de Mauria, género que Benthan y Hooker (1852) incluyeron en la tribu Anacardineae de la familia Anacardiaceae; Marchan (1869) lo reconoció como una sección del género Sorindeia Dup-Th y redujo a su sinonímia a las siete especies descritas hasta entonces. Engler (1876), al revisar las Anacardiaceae, aceptó el concepto anterior, ubicando a Sorindeia dentro de la tribu Rhoidea pero posteriormente (Engler 1883) el mismo lo reconoció como un género aparte, al igual que Smith (1897), Loesner (1906), Herzog(1909), Standley (1930, 1937, 1947), Barkley (1942, 1947, 1957) y Cuatrecasas (1955). Después del trabajo de Engler (1883) sólo se ha realizado la presente revisión del género (Rentería, 1988).

Las características principales de Mauria son: árboles o arbustos, hojas simples o compuestas, flores completas y prefectas, con dos veces el número de estambres de pétalos, fruto drupa con endocarpio semejante a pergamino, ovario superior con un celda, semilla suspendida y nectario entre los estambres y el ovario.

Esta publicación es resultado del proyecto de investigación denominado como Mauria de Colombia, que se realizó financiado por Colciencias (970891-82), Jardines Botánicos de Missouri y Medellín y la Universidad de Antioquia donde se realizó un arduo trabajo de campo, se ha utilizado toda la información disponible en morfología interna y externa, química, dendrología y palinología, con el auxilio de las técnicas modernas tales como el microscopio electrónico de barrido y el computador; se ha efectuado además, un cuidadoso trabajo de campo requisito indispensable para facilitar la caracterización del género y sus especies, establecer su distribución, conocer su ecología, cuantificar la relación entre las especies e interpretar su filogenia.

Esta investigación constituye una contribución al estudio de la flora de Colombia no sólo por los datos útiles que aporta desde el punto de vista taxonómico, sino porque permite señalar, por una parte que el estudio farmacológico de las especies del género es deseable en vista de sus reconocidas propiedades alergénicas y, por otra parte, que existe la posibilidad de la explotación de su corteza y hojas para la extracción de taninos y los frutos como materia prima para la producción de un posible perfume.

Mauria amaliae constituye una de las tres especies nuevas encontradas para la flora colombiana. Antes de esta investigación Colombia sólo reportaban 11 especies, hoy son 14 .

Mauria amaliae Renterias sp.nov. Tipo: Colombia, Magdalena. Sierra Nevada de Santa Marta, Mar 1852, Schlim 797 fl.! (Holotipo BR!; Isotipos G!, K!)

Diagnosis. Arbor vel arbuscula, 3-8 m alta, resina odorata, folia, in spiralibus simplicia, obovata vel ellipptico -lanceolata, habentia pubescentes axillas nervorum infra; superficies ceracea foliorum filis dispersis posita. stomata amphipericitica, 4-5 cellulis subsidiariis, duae margines circum stomata, pollen parvum, oblato -sphaerideum, tricholporatum, exinium striatum.

Descripción. Arbusto o árbol de 3-8 m de altura, corteza glabra con escamas. Hojas espiraladas, simples, elíptico-lanceoladas u obovadas, pecíolo 0.5$1.5 \mathrm{~cm}$ de longitud, subterete, puberulo. Lámina 2.5 $12 \mathrm{~cm}$ de longitud por $1.5-5.5 \mathrm{~cm}$ de ancho, chartácea, ápice retuso, base atenuada, margen entero, haz glabra, envés pubescentes en las axilas de las nervaduras; costa media prominente o impresa por la haz, 


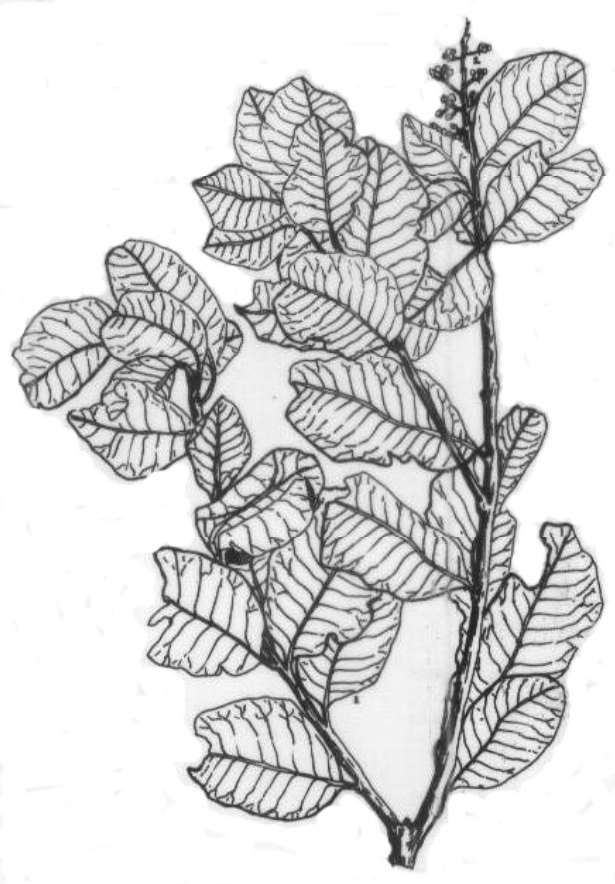
Figura 2. Mauria amaliaeRentería var. amaliae
(Schlim 797, BR). Hábito. Reducción 1/4.

prominula por el envés, nervaduras secundarias 7-14 pares semiparalelas, prominentes o impresas en ambas caras. Inflorescencia terminal o axilar, panicula pubescentes; brácteas ovadas, coriáceas, ciliadas, 1-2 mm de longitud. Flor pedicelada, receptáculo plano, estivación valvar, cáliz verde, pubescente externamente, urceolado-obtuso, lóbulos 5; corola rosada, pétalos 5 , libres, alternas con sépalos ovados, 2$3 \mathrm{~mm}$ de longitud,1-2 $\mathrm{mm}$ de ancho, coriáceos, ápice agudo, base truncada; estambres 10, libres, filamento trígono, 1-1.5 $\mathrm{mm}$ de longitud; anteras ovadas-oblongas, $0.5 \mathrm{~mm}$ de longitud, dorsifijas; ovario oblongo, $0.5-1 \mathrm{~mm}$ de longitud; estilo circular, pequeño, estigma 3-4, en forma de corona, laterales; nectário amarillo, con 10 crenaciones. Fruto oblongo, $0.7-1 \mathrm{~cm}$ de longitud por 0.4-0.6 cm de ancho, sépalos, estambres, nectários y estigma persistentes.

Los granos de polen (Figuras 4 A, B), son pequeños (21.8 micras, en vista ecuatorial), oblado esferoidal, isopolar con simetría bilateral, tricolporado (poro de 2.5 micras por 1.3 micras), la exina es estriada; la relación $\mathrm{P} / \mathrm{E}$ es igual a 0.91 . Estos granos de polen son muy semejantes a los de géneros muy afines como: Lithraea (Anzoátegui, 1971) y Rhus (Heinsch, 1940).

Etimología. Esta especie se ha dedicado a una botánica colombiana que colaboró mucho durante esta investigación Amalia Cruz Cecilia Estrada.

Distribución. La especie se conoce en Venezuela y Colombia (Sierra Nevada de Santa Marta y en el Departamento del Huila). Patrón de distribución alopátrica y endémicas de

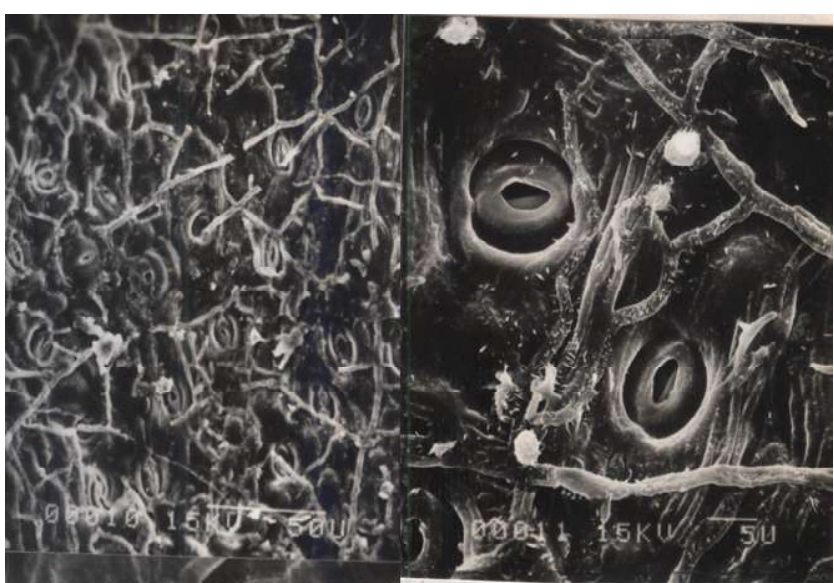

Figura 3. Fotografía al microscopio electrónico de barrido de Mauria amaliae Renteria var. amaliae (Schlim 797, BR). A. Superficie cerosa del envés con hilos dispersos (X 480). B. Hilos de cera y estomas (X 1900). C. Estomas anfipericiticos con los dos rebordes periestomatales (X 3600).

cada departamento. El tipo de polinización de la especie es entomófila; el síndrome que presenta sus poblaciones son flores pequeñas, color amarillas, no vistosas, pétalos pequeños, nectario presente, aromas fuertes, estambres y pistilos expuestos. Su hábitat preferido son los lugares cerca de quebradas, fuentes de agua y zonas anteriormente bañadas por éstas y en pequeñas pendientes en suelos de cordilleras.

El análisis del material disponible, permite concluir, que existen dos variedades.

Clave para las subespecies de Mauria amaliae

1. Árbol, hojas obovadas, ápice retuso, nervadura secundaria impresa tanto en la haz como en el envés .........1a. subespecie amaliae

1. Arbusto, hojas elíptico-lanceoladas, ápice acuminado, envés con nervadura primaria con dos surcos, nervadura secundarias prominentes tanto en la haz como en el envés............1b. subespecie huilae

1a. Mauria amaliae subespecie amaliae Rentería nov. Subespecie Figuras 1, 2, 3, 4

Tipo: Colombia, Magdalena. Sierra Nevada de Santa Marta, Mar 1852, Schlim 797 fl.(Holotipo BR!; Isotipos G!, K!)

Diagnosis: arbor, folia obovata, apez retuso, nervatio secundária impressa tan supra quam infra folia. Árbol de 5-8 $\mathrm{m}$ de altura, hojas obovadas, ápice retuso, nervaduras secun- 


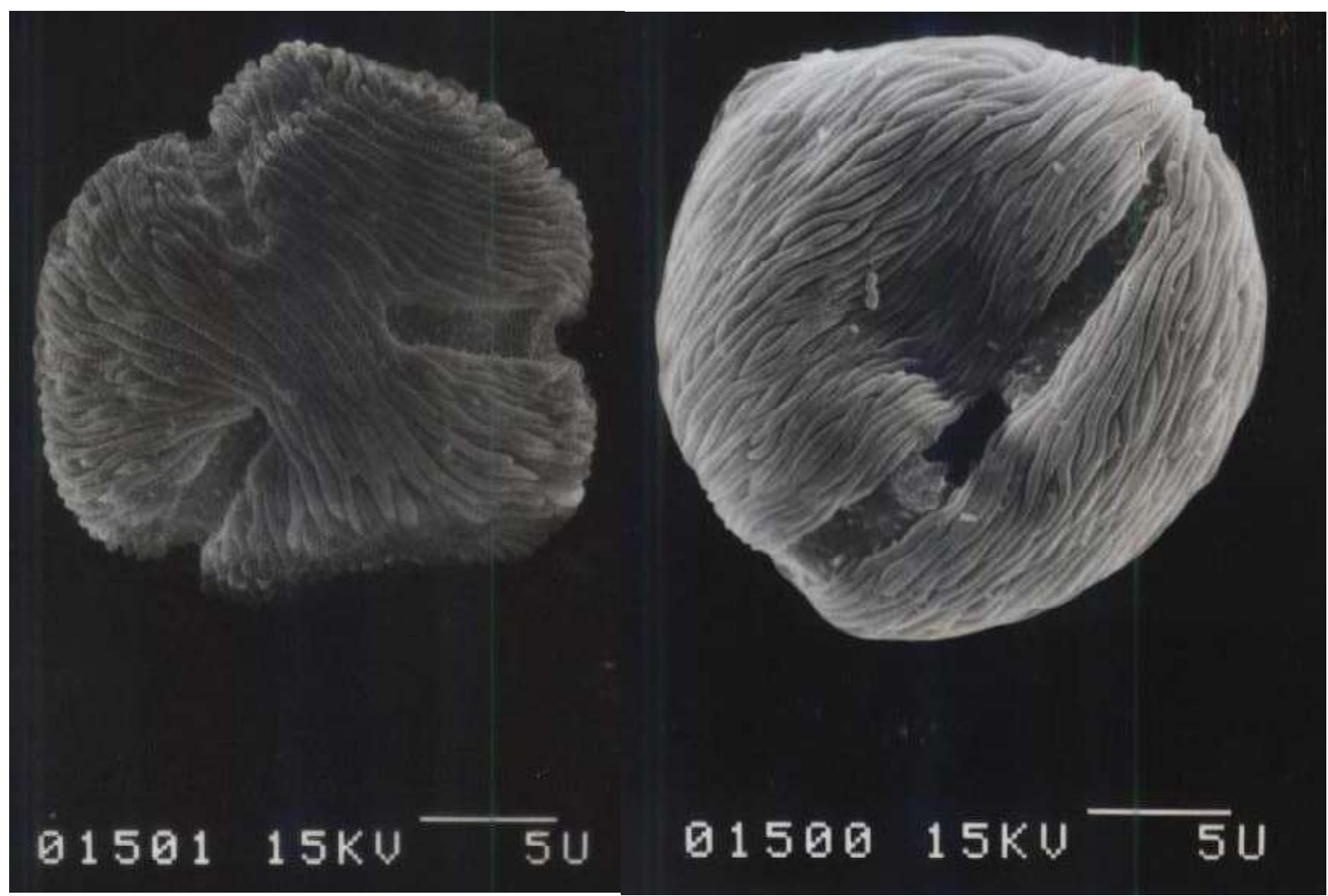

Figura 4. Mauria amaliaeRenteria var. amaliae(Schlim 797, BR). A. Vista polar mostrando la naturaleza tricolporada y la exina estriada (X 1500). B. Vista ecuatorial mostrando el poro y el colpo (X 1500)

darias impresas tanto en el haz como en el envés.

Distribución: la variedad tipo crece en el estado de Mérida (Venezuela) y en la Sierra Nevada de Santa Marta.

Paratipos. Colombia, Magdalena: Sierra Nevada de Santa Marta, sin fecha, Purdie s.n. fl. (K!).Venezuela; Estado de Mérida Distrito libertador, Caserío el Chabá, 14 de Dic 1961, Ruiz-Terán $826 \mathrm{fl}$ (MO!).

Esta variedad se puede reconocer en el campo por ser árbol de 5-8 $\mathrm{m}$ de altura, 15-20 $\mathrm{cm}$ de diámetro a la altura del pecho(DAP), con látex resinoso; algunas de las flores presentan 4 estigmas, sus hojas son simples con pubescencia en las axilas de las nervaduras del envés. Posee estomas anfipericiticos con 4-5 células subsidiarias; las células de guarda son hundidas y con ornamentación estriada, con dos áreas gruesas pericrinales, apertura del estoma es casi ovalada y mide de 4-5 micras (Figura 3 A, B, C). Los granos de polen (Figura 4 A, B), son pequeños (21.8 micras, en vista ecuatorial), oblado esferoidal, isopolar con simetría bilateral, tricolporado (poro de $2.5 \times 1.3$ micras), la exina es estriada; la relación $\mathrm{P} / \mathrm{E}$ es igual a 0.91 . Estos granos de polen son muy semejantes a los de géneros muy afines como Lithraea (Anzoátegui, 1971) y Rhus (Heinsch, 1940). Además la superficie serosa de las hojas está formada por hilos dispersos
(Figuras 3 A, B, C).

1b. Mauria amaliae subespecie. huilae Rentería., nov. subespecie (Figuras 1, 3, 4, 5)

Tipos: Colombia; Huila: San Agustín, 19 Abr 1944, Litte 7695 Fl y Fr (Holótipo COL!; Isótopos US! )

Diagnosis. Arbuscula, folia eliptico-lanceolata, apex acuminatus, infra folium nervatione primaria duplicibus sulcis, nervationes secundariae prominentes tam supra quam infrra folia. Arbusto de 3-4 m de altura, hoja elíptico-lanceolada ápice acuminado envés con nervadura principal con dos surcos; nervaduras secundarias prominentes tanto en el haz como en el envés.

Etimología. Esta subespecie se dedica al departamento del Huila.

Distribución. Ha sido coleccionada en San Agustín (Colombia) y en los estados de Mérida y Táchira (Venezuela) entre 2000 y $3000 \mathrm{msnm}$.

Paratipos. Venezuela; estado de Mérida: Monte de Zerpa, 19 de Sep de 1942 Lasser 340 fl(S US, VEN); la Muery, sep1942, lasser $537 \mathrm{fl}(\mathrm{US}, \mathrm{VEN}$ ); timotes, 23 de enero 1928, Pitter 12702 FL(G, NY, US, VEN); estado de Táchira, la grita en el pregonero, 9 oct 1965, Bernardi $10979 \mathrm{FL}(\mathrm{G})$; portachuelo, la grita, 13 de may 1971, Ferrari 909 FL(NY). 


\section{Bioetnia Volumen 5 No 2 (julio-diciembre), 2008}

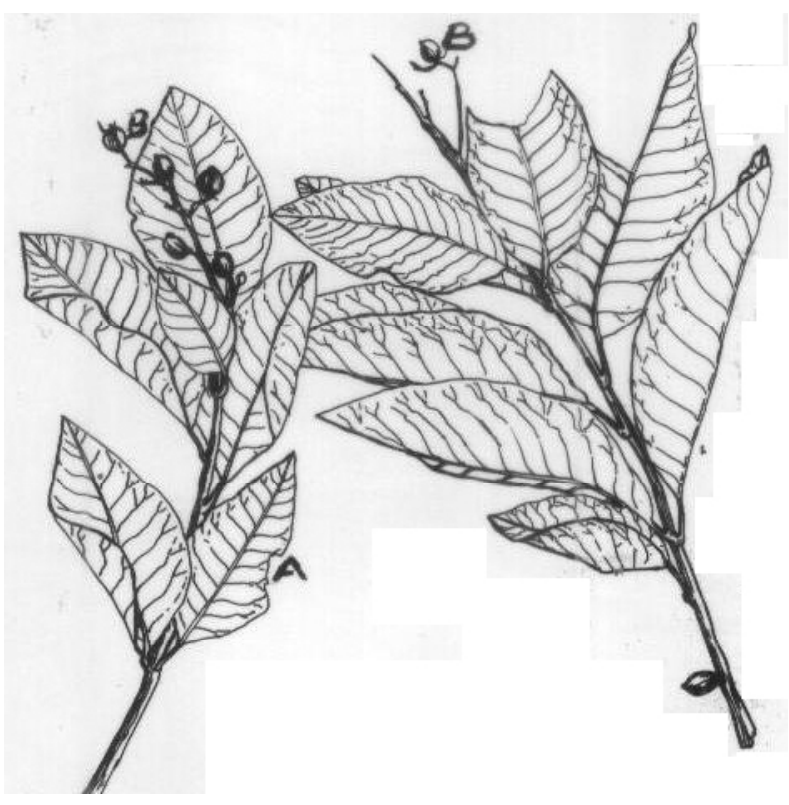

Figura 5. Mauria amaliaeRenteria var. huilaea (Litte 76959). A. Hábito. B. Frutos. Reducción 1/4

NV. Caunche (Mérida, Venezuela). Se reconoce esta variedad en el campo por ser un arbusto con altura máxima de 4 $\mathrm{m}$, sus hojas son elíptico-lanceoladas con ápice acuminado, inflorescencia terminal y axilar, muy ramificada y 3 estigmas.

Observaciones taxonómicas. El estudio fenético se realizó con el objeto de utilizar las técnicas numéricas para determinar la relación de similitud entre las especies con base en los estados de los caracteres siguiendo a Crisci \& López Armengol(1983), utilizando las técnicas con base a Sneath \& Sokal (1973), Sokal(1961), Cain y Harrison(1958), Michener \& Sokal (1957) y se procesaron los datos con los programas del NT-SYS (Rohlf \& Kishpaugh, 1971); las matrices fueron estandarizadas. La técnica que representa mejor las relaciones de distancia entre las especies es la de ligamento promedio (Figura 6) en la cual se observan claramente dos grupos bien definidos, con una escasa distorsión, ya que el coeficiente de correlación cofenética dio un valor de 0,81 Crisci \& López Armengol (1983).Uno de ellos se puede denominar como de las hojas simples donde encontramos a Mauria amaliae, Mauria simplicifolia y Mauria heterophylla entre las cuales se pueden subdividir en dos grupos y los caracteres que contribuyen para este fin son la pubescencia de la hoja y el estado del pólen (tamaño, exina y forma). M. amaliae forma un subgrupo y se caracteriza por poseer pólen pequeño, exina estriada, envés pubescente, mientras el otro sugrupo esta conformado por M. simplicifolia y M. heterphylla con pólen mediano, exina foveolada y envés glabro. El otro grupo se forma por las 11 especies restantes que poseen hojas compuestas, con cuatro subgrupos bien definidos.
El estudio cladístico se basó en 47 caracteres a los cuales se les determinó la polaridad. Es decir, el estado plesiomórfico o apomórfico, (Hennig, 1968) lo que dice si es primitivo o avanzado utilizando como géneros afines a Lithraea y Schinus (Engler, 1876; Berkley, 1947) tanto por sus características morfológicas como por su origen (son taxa originarios de los Andes). De las cuatro opciones analizadas mediante el programa Wagner-78 (Farris, 1970) se seleccionó a M. birringo como la especie más primitiva por ser una de las que posee mayor cantidad de estados plesiomóficos (36) y por contribuir a establecer mejor las relaciones entre especies y los caminos evolutivos seguidos por los caracteres, los cladogramas (Rentería, 1988) muestra una gran similitud con el fenograma, es decir, se forma 4 grupos, uno de ellos, el más evolucionado está conformado por Mauria amaliae,, Mauria heterophylla,Mauria peruviana y Mauria simplicifolia, todas de hojas simples, excepto, Mauria heterophylla, que presentan hojas simples y compuestas, es decir, heterofilia, la especie más distante de este grupo es Mauria peruviana (distancia 34,6) con 6 apomorfías y 4 reversiones. La especie que dio origen a este grupo es de hojas compuestas, con pubescencia y árboles ( $M$. ferruginea) que evolucionaron a especies con hojas simples, glabras y arbustos (Rentería, 1988). Cuando se realizó el estudio químico de algunas espécies de Mauria, se encontró en el mismo sugrupo a $M$. ferruginea y $M$. simplicifolia, lo cual corrobora lo anterior) al analizar a Mauria amaliae, observamos que es la segunda especie más primitivas de este grupo, que presente 4 apomorfías y 2 reversiones; se encuentra entre $M$. Gentrii y $M$. heterophylla la primera de hojas simples y la segunda presenta heterofilia (Rentería, 1988).

\section{AGRADECIMIENTOS}

Se reconoce y agradece la valiosa asesoría científica de los doctores Enrique Forero, U. Nacional de Colombia, Bogotá y Alwin H. Gentry, Missouri Botanical Garden, qepd. Jorge Víctor Crisci, Universidad de la Plata, Argentina), sus colaboradores y al Centro de Estudios Superiores para el Procesamiento de la Información, Walter Lewis, U. de Washington, St. Louis Missouri y su grupo de técnicos por facilitarme su laboratorio y equipos para realizar el análisis polínico y de estomas en el microscopio de luz y electrónico de barrido. A Peter Raven por proporcionarme la oportunidad de permanecer 6 meses en el Jardín Botánico de Missouri y utilizar su laboratorio, biblioteca, toma de fotografías electrónicas durante mi permanencia en dicha institución. También son merecedores de reconocimiento el profesor Roberto Jaramillo, el doctor Alvaro Fernández-Pérez (qepd), la Ingeniera Agrónoma Olga Salazar de Benavides, Licenciado Edgar Bonilla, el Ingeniero Agrónomo Carlos Nates y el doctor Tom Croat, quienes colaboraron en el trabajo de campo; al profesor 


\section{Nueva especie de Mauria (Anacardiaceae) para Colombia. E Rentería}

Fernando Echeverri y la Bióloga Blanca Luz Mejía por su colaboración al realizar un estudio preliminar de la química de algunas especies de Mauria. Para la realización de este trabajo se contó con la colaboración de las siguientes entidades Colciencias proyecto $\mathrm{N}^{\circ}$ 97089-1-82, Jardín Botánico de Medellín, Jardín Botánico de Missouri, U. de Antioquia, U. Nacional de Colombia, U. de la Plata, Argentina, a quienes expreso mis sinceros agradecimientos, lo mismo para los directores y curadores de los siguientes herbarios por facilitarme los ejemplares (incluyendo los tipos): BM, BR, C, COL, F, G, K, JUAM, MA, MEDEL, MO, NY, P, S, y W. al grupo de Investigación de Biosistemática, al Semillero de Sistemática de Flora Chocoana y a la Universidad Tecnológica del Chocó por alentarme a realizar esta publicación.

\section{LITERATURA CITADA}

Anzoátegui, L. 1971. Polen de la Anacardiaceae del N.E. de la Argentina. Ameghiniana. 8 (3-4): 329-40.

Barkley, F.A. 1942. A key genera of the Anacardiaceae. Amer Midl Nat. 28: 46574.

Barkley, F.A 1947. Specimen of Mauria. Bull Torr Bot Club. 74 (1): 77-80.

Barkley, F.A 1957. Generic key to the Sumac family (Anacardiaceae). Llodia. 20 (4): 255-65.

Bentham, G., Hooker J.D. 1862. Genera Plantarum. 1: 415-28.

Cain, A.J., Harrinson G.A. 1958. An analysis of the taxonomist's judgement of affinty. Procced. Zoological Soc London. 131: 85-98.

Crisci, J.V., López-Armengol M.F. 1983. Introducción a la teoría y práctica de la taxonomía numérica. Monografía. Washington, DC: OEA.

Cuatrecasas, J. 1955. Taxonomic notes on neotropical tres. Tropical Woods. 101: $12-3$.

Endlicher, S. 1836. Genera Plantarum. 1: 1130-6.

Engler, A. 1876. Anacardiaceae. En Mart. Fl. Bras. 12 pt. 390.
Engler, A. 1883. Anacardiaceae, en C. De Candolle. Monogr Phanner. 4: $325-$ 31.

Farris, J.S. 1970. Methods for computing Wagner trees. Syst Zool. 19: 87-92.

Heimsch, C. 1940. Word anatomy and pollen morphology of Rhus and allied genera. J Arnold Arb. 21 (3): 279.

Hennig, H. 1968. Elementos de sistemática filogenética. Editorial Universidad de Buenos Aires.

Herzog, T 1909. Anacardiaceae. Fedde Rep. 7: 60-1.

Kunth, C. S. 1824. Terebinthacearum genera. Ann Sci Nat. 2 (36): 333-66.

Kunth, C. S. 1825. Kunh in Humboldt \& Bonpland. Nov. gen. sp. 2: 1.

Lindley, J. 1831. Introduction to the natural system of botany. 125-7.

Loesner, Th. 1906. Anacardiaceae Andinae. Bot Jahrb. 37: 570-5.

Macbride, J. F. 1951. Flora of Perú. Anascardiaceae. Field Mus Nat Hist Bot. 13: $238-58$.

Marchand, L. 1869. Revision du grupe des Anacardiaceés. Paris. p. 165.

Michener, C. D. Sokal, R. 1957. A quantitative approach to a problem in classification. Evolution. 11: 130-62.

Poeppig, E. F. 1845. Anacardiaceae. Poeppig \& A. Engler Nov. Gen. et sp. 3: 77.

Rentería, E. 1988. Resivión de las especies colombianas del género Mauria Kunth. Tesis de Maestría, Intituto de Ciencias Naturales, Facultad de Ciencias, Universidad Nacional de Colombia, Bogotá, DC.

Rohlf, F.J., Kispaugh J., Kirk D.. 1971. NT-SYS.Numerical taxonomy system of multivariate statical programs. Techn Rep. State University of New York at Stony Brook, New York; 87 pp.

Sneath, P.H., Sokal, R. 1973. Numerical taxonomy. Principles and practice of numerical classification. San Francisco: W.H. Freeman and Co.

Sokal, R. R. 1961. Distance as measure of taxonomy similarity. Syst Zool 10: 70 .

Standley, P. C. 1930. The woody plants of Honduras. J Arnold Arb. 11: 33.

Standley, P. C. 1937. Flora of Costa Rica. Anacardiaceae. Field MustNat Hist Bot. 18: 627-8.

Standley, P. C. 1947. Flora of Guatemala. Anacardiaceae. Field Mus Nat Hist. 24 (6): 177-95.

Tulasne, L.R. 1846. Plantes Nouvelles de la Colombia. Anacardiaceae. Ann Sci Nat Bot Ser. 3(6): 362-8.

Turczaninow, N. 1858. Anacardiaceae. Bull Soc Nat Moscow. 31 (1): 467-8. 